\title{
COMPOSIÇÃO E ATIVIDADE ANTIOXIDANTE DAS DIFERENTES PARTES DO ALHO-PORÓ (Allium porrum) PRÉ E PÓS COCÇÃO ${ }^{1}$
}

\author{
COMPOSITION AND ANTIOXIDANT ACTIVITY OF THE \\ DIFFERENT PARTS OF LEEK (Allium porrum) PRE-AND POST-COOKING
}

\author{
Barbara Damin², Cátia Santos Branco ${ }^{3}$, Elizete M. P. Facco ${ }^{4}$ e Gabriela Chilanti ${ }^{5}$
}

\section{RESUMO}

O alho-poró (Allium porrum) é uma hortaliça que pertence ao gênero Allium, juntamente com a cebola, o alho, cebolinha, dentre outros. É consumido em nível mundial como ingrediente, tempero, condimento e também como medicamento. Seu talo (bulbo) é de cor branca, muito apreciado em preparações culinárias incluindo sopas, molhos, risotos e saladas. As folhas verdes, no entanto, são geralmente descartadas. O gênero Allium é caracterizado por possuir baixa densidade calórica, além de ser rico em luteína, $\beta$-caroteno, vitaminas $\mathrm{C}$ e $\mathrm{E}$, além de polifenóis. Embora seja muito apreciado na culinária, os dados acerca da composição química e atividade antioxidante do alho-poró são escassos. O objetivo deste trabalho foi analisar dois cultivares de alho-poró Atal (A) e Lancelot (B) quanto a composição centesimal, conteúdo fenólico total e atividade antioxidante, bem como a sua estabilidade sob diferentes processos térmicos. Os resultados mostraram que os teores de umidade, cinzas e lipídeos foram maiores no cultivar $\mathrm{B}$, enquanto que teores de proteína e carboidrato foram maiores no cultivar A. Polifenóis totais e atividade antioxidante também foram maiores no cultivar B, e com diferenças significativas entre bulbo e folhas, onde os valores foram maiores nas folhas. Houve redução significativa de fenólicos totais e atividade antioxidante nas amostras quando cozidas e aumento, quando refogadas. Com o presente estudo pode-se concluir que as folhas são importantes fontes de nutrientes e antioxidantes, sendo importante incentivar o consumo dessa hortaliça como um todo, sem desperdício de alimento e de nutrientes.

Palavras-chave: Compostos Fenólicos, Nutrientes, Aproveitamento Integral dos Alimentos.

\section{ABSTRACT}

Leek is a vegetable that belongs to the genus Allium, along with onion, garlic, chives, among others. The leek is consumed worldwide as an ingredient, seasoning, condiment, and also as a medicine. Its stem (bulb) is white, which has been extensively used in cooking including soups, sauces, risottos, and salads. Green leaves, however, are usually discarded. The genus Allium is characterized by low caloric density besides being a rich source of lutein, $\beta$-carotene, vitamins $C$ and $E$, and polyphenols. Although very popular in cooking, data on the chemical composition and antioxidant activity of leeks are scarce. Two cultivars of leeks Atal (A) and Lancelot (B) were analyzed for macronutrients, phenolic content, antioxidant activity, as well as their stability under different thermal processes. The results showed that humidity, ash, and lipid contents were higher in cultivar B, whereas protein and carbohydrate levels were higher in cultivar A. Total phenolics and antioxidant activity were also higher in cultivar $B$, and with significant differences between bulb and leaves, where values

\footnotetext{
${ }^{1}$ Artigo Científico.

${ }^{2}$ Acadêmica do Curso de Nutrição - Universidade de Caxias do Sul - UCS. E-mail: bdamin@ucs.br

${ }^{3}$ Colaborador. Docente da Área do Conhecimento de Ciências da Vida. Laboratório de Estresse Oxidativo e Antioxidantes, Instituto de Biotecnologia - Universidade de Caxias do Sul - UCS. E-mail: csbranc1@ucs.br

${ }^{4}$ Colaborador. Docente da Área do Conhecimento de Ciências da Vida. Laboratório de Bromatologia e de Microbiologia de Alimentos - Universidade de Caxias do Sul - UCS. E-mail: empfacco@ucs.br

${ }^{5}$ Orientador. Docente do Curso de Nutrição - Universidade de Caxias do Sul - UCS. E-mail: gchilant@ucs.br
} 
were higher in leaves. There was a significant reduction of total phenolics and antioxidant activity in cooked samples and it increased when fried. With the present study, it can be concluded that leaves are significant sources of nutrients and antioxidants, and it is important to encourage the consumption of this vegetable as a whole, without wasting food and nutrients.

Keywords: Phenolic Compounds, Nutrients, Whole Utilization of Foods.

\section{INTRODUÇÃO}

Allium porrum é uma hortaliça conhecida popularmente como Alho-poró, Alho-francês, Porro-bravo, dentre outras sinonímias. Pertence à família Alliaceae e ao gênero Allium, juntamente com alho (Allium sativum) e cebola (Allium cepa), entre outras espécies do gênero (LANZOTTI, 2006; SHARIFI-RAD et al., 2016). É um vegetal amplamente cultivado no mundo, e tem origem Europeia. Comparado a outras culturas do gênero, o alho-poró se adapta bem a altas temperaturas, embora sua temperatura ideal de cultivo seja de $20{ }^{\circ} \mathrm{C}$ (CLERCQ et al., 2003).

A espécie Allium ampeloprasum var. porrum L. é uma planta herbácea bulbosa e perene, cultivada e usada não somente como alimento, mas também como medicamento (CORRÊA, 1978). Na culinária, o talo (bulbo) é a parte mais consumida, sendo utilizada em uma variedade de preparações (PAK et al., 2014), enquanto que as folhas verdes são subutilizadas. Na medicina tradicional brasileira tem sido relatado o uso dos bulbos do alho-poró como infusão para tratar sintomas de doenças inflamatórias, e quando esmagado, pode ser utilizado para tratar estágios iniciais de tosse. O suco fresco também é utilizado como estomático e antiespasmódico, além de ser conhecido por possuir propriedades digestivas (CORRÊA, 1978). Apesar de seus usos empíricos e seu consumo na culinária, são escassos os estudos científicos acerca dessa planta.

Dados experimentais e epidemiológicos demonstram que o consumo dos vegetais do gênero Allium reduz os riscos de câncer de próstata, mama, estômago e também de colo retal (HSING et al., 2002; NICASTRO et al., 2015; ASEMANI et al., 2019). Outras propriedades atreladadas ao consumo de plantas desse gênero incluem atividade antibacteriana, antiviral, imunoestimulatória, melhoradora do perfil lipídico e antioxidante (KOTHARI et al., 2019).

As propriedades farmacológicas relatadas para as espécies do gênero Allium são atribuídas à presença de diversos compostos bioativos em suas diferentes partes. Dentre os compostos destacam-se saponinas, frutanos, fruto-oligossacarídeos, compostos organosulforados e os polifenóis (LANZOTTI, 2006; SHARIFI-RAD et al., 2016; KOTHARI et al., 2019). Especificamente, no alho-poró foi descrita a presença de $\beta$-caroteno, luteína, além da vitamina C (ácido ascórbico) (PROTEGGENTE et al., 2002).

Tendo em vista os inúmeros benefícios associados ao consumo de plantas do gênero Allium, e a escassez de estudos específicos sobre o alho-poró, o presente estudo teve como objetivo avaliar a composição centesimal, atividade antioxidante e conteúdo fenólico total do bulbo (parte nobre) e das 
folhas de dois cultivares de alho-poró in natura e após passar por processos térmicos (cozimento e quando refogado).

\section{MATERIAL E MÉTODOS}

Estudo experimental em análise de alimentos. Foram utilizadas quatro amostras para as análises, sendo duas do cultivar Atal que é caracterizada por bulbo mais longo e folhas de cor verde claro (Figura $1 \mathrm{~A}$ ); e duas do cultivar Lancelot, que apresenta bulbo mais curto e folhas de cor verde escuro (Figura 1 B). As amostras foram colhidas no dia anterior das análises, no distrito de Santa Lúcia do Piaí, município de Caxias do Sul/RS. O preparo das amostras consistiu na separação das folhas do bulbo, e em seguida as partes foram processadas em um mixer, e então homogeneizadas. Posteriormente, foram submetidas à diferentes formas de preparo, sendo in natura ou após cocção (cozido ou refogado). As amostras foram preparadas em laboratório de modo a simular o processamento doméstico da hortaliça: a) as mesmas foram refogadas utilizando uma colher de café de óleo de soja (6 g) até dourar; ou b) cozidas, através de fervura por 25 minutos, simulando o preparo de um caldo. A descrição detalhada da preparação e processamento das amostras está descrita na Figura 2.

Figura 1. Alho-poró cultivar Atal (A) Lancelot (B).

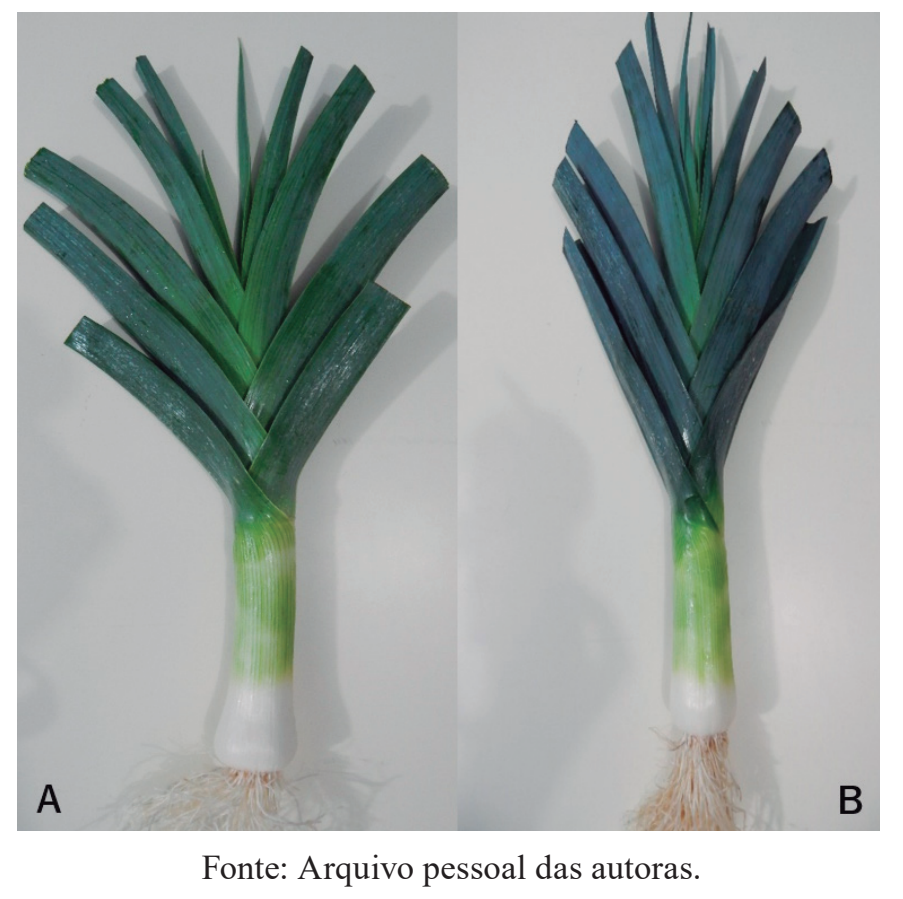

A composição centesimal das amostras in natura (a-c) foi realizada de acordo com metodologias descritas pelo Instituto Adolfo Lutz (IAL, 2008) e os resultados foram expressos em g/100g: a) o teor de umidade foi analisado pelo método da estufa a $105^{\circ} \mathrm{C}$ até peso constante; b) a determinação de cinzas foi realizada pelo método da incineração a $600{ }^{\circ} \mathrm{C}$ em mufla; c) o teor de proteína foi quantificado através do método de micro Kjeldhal, com fator de conversão de 6,25. Os lipídeos foram obtidos pela 
extração com a combinação de solventes à frio e quantificados por gravimetria (BLIGH E DYER, 1959). Os carboidratos foram estimados por diferença subtraindo-se de 100 os valores obtidos para umidade, proteínas, lipídios, cinzas. Para determinação do valor energético foram utilizados os resultados em gramas obtidos para carboidratos, proteínas e lipídeos, somados aos fatores de conversão específicos, a saber: carboidratos - 4kcal/g; proteínas - 4kcal/g; lipídeos -9kcal/g, de acordo com AOAC (2011).

Figura 2. Fluxograma mostrando as etapas de preparação e processamento das amostras.

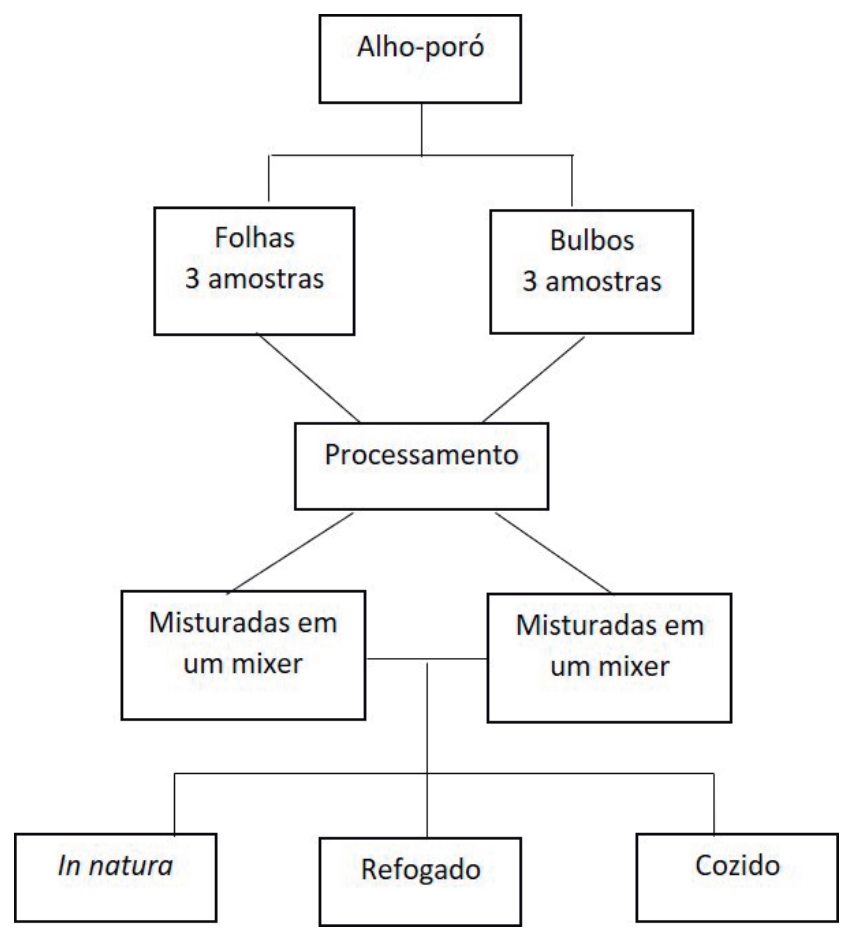

Fonte: As autoras.

A quantificação dos compostos fenólicos totais foi determinada de acordo com o método colorimétrico de Folin-Ciocalteu, com modificações (SINGLETON E ROSSI, 1965). Os resultados foram expressos em mg equivalentes de ácido gálico (EAG)/100g de amostra. A determinação da atividade antioxidante foi realizada através da medida do radical ABTS, através da metodologia descrita por Re et al. (1999). Os resultados foram expressos em mM equivalentes de trolox (TE)/g.

Os testes foram realizados em triplicata e os resultados foram expressos em média \pm desvio padrão. Os dados foram comparados por análise de variância (one-way ANOVA) e pós-teste de Tukey, considerando nível de significância inferior a $5 \%(p<0,05)$, utilizando o software Prism GraphPad ${ }^{\circledR}$ (versão 5.0.1.334).

\section{RESULTADOS E DISCUSSÃO}

Os resultados da composição nutricional das diferentes partes do alho-poró estão apresentados na Tabela 1. Foi possível observar diferenças significativas em praticamente todos os parâmetros 
avaliados quando comparadas as folhas ao bulbo. O teor de umidade obtido nas amostras de bulbo variou de $84,15 \pm 0,12 \mathrm{~g} / 100 \mathrm{~g}$ (cultivar B) a 86,58 $\pm 0,19 \mathrm{~g} / 100 \mathrm{~g}$ (cultivar A). A folha apresentou o maior teor de umidade com pequena diferença, variando entre 90,36 $\pm 0,04 \mathrm{~g} / 100 \mathrm{~g}$ (cultivar A) e $90,76 \pm 0,06 \mathrm{~g} / 100 \mathrm{~g}$ (cultivar B).

Em estudo realizado por Shad et al. (2016) que avaliou a composição nutricional e atividade antioxidante de plantas medicinais subutilizadas, como alho-poró, salsão, entre outras, os valores de umidade encontrados foram de 50,58 g/100 g no alho-poró, valor inferior aos encontrados no presente estudo, e 70,98 g/100 g no salsão, resultado aproximado, mas também inferior ao do encontrado nas amostras deste estudo.

Storck et al. (2013) avaliaram separadamente a composição de talos, folhas, cascas e sementes de diferentes vegetais, exceto alho-poró. Os valores aproximados aos de umidade descritos em nosso estudo foram encontrados para folhas de cenoura $(86,9 \mathrm{~g} / 100 \mathrm{~g})$, partes nobres da beterraba $(86,0 \mathrm{~g} / 100 \mathrm{~g})$, cascas de batata $(85,1 \mathrm{~g} / 100 \mathrm{~g})$ e partes nobres do brócolis $(91,2 \mathrm{~g} / 100 \mathrm{~g})$.

Tabela 1 - Composição centesimal (g/100 g) de bulbo e folhas do alho-poró (Allium porrum).

\begin{tabular}{ccccc}
\hline & \multicolumn{2}{c}{ Bulbo } & \multicolumn{2}{c}{ Folha } \\
\hline Cultivares & A & B & A & B \\
\hline Umidade & $86,58 \pm 0,19^{\mathrm{b}}$ & $84,15 \pm 0,12^{\mathrm{b}}$ & $90,36 \pm 0,04^{\mathrm{a}}$ & $90,76 \pm 0,06^{\mathrm{a}}$ \\
\hline Cinzas & $0,74 \pm 0,01^{\mathrm{b}}$ & $0,75 \pm 0,01^{\mathrm{c}}$ & $0,83 \pm 0,01^{\mathrm{b}}$ & $1,14 \pm 0,01^{\mathrm{a}}$ \\
\hline Proteína & $1,60 \pm 0,01^{\mathrm{a}}$ & $1,61 \pm 0,01^{\mathrm{a}}$ & $1,57 \pm 0,04^{\mathrm{a}}$ & $1,58 \pm 0,05^{\mathrm{a}}$ \\
\hline Extrato etéreo & $0,24 \pm 0,01^{\mathrm{b}}$ & $0,25 \pm 0,01^{\mathrm{b}}$ & $0,54 \pm 0,02^{\mathrm{a}}$ & $0,54 \pm 0,01^{\mathrm{a}}$ \\
\hline Carboidrato & $10,08 \pm 0,65^{\mathrm{b}}$ & $12,19 \pm 0,06^{\mathrm{a}}$ & $6,22 \pm 0,75^{\mathrm{c}}$ & $7,81 \pm 1,24^{\mathrm{bc}}$ \\
\hline Energia (Kcal) & $46,76 \pm 2,98^{\mathrm{b}}$ & $61,62 \pm 0,63^{\mathrm{a}}$ & $36,04 \pm 2,89^{\mathrm{c}}$ & $48,09 \pm 4,55^{\mathrm{b}}$ \\
\hline
\end{tabular}

Legenda: Os valores apresentados são resultantes da média de três repetições \pm desvio padrão. Em cada linha, letras diferentes diferem estatisticamente $(\mathrm{p}<0,05)$.

O teor de cinzas reflete a quantidade de minerais presentes em um alimento. Nesse parâmetro, o bulbo apresentou variação de $0,74 \pm 0,01 \mathrm{~g} / 100 \mathrm{~g}$ (cultivar A) a 0,75 $\pm 0,01 \mathrm{~g} / 100 \mathrm{~g}$ (cultivar B), enquanto que a folha apresentou maior quantidade de cinzas, variando entre $0,83 \pm 0,01$ (cultivar A) a 1,14 $\pm 0,01 \mathrm{~g} / 100 \mathrm{~g}$ (cultivar B) (Tabela 1). O teor de cinzas encontrado por Shad et al. (2016) foi 3,97 g/100g, cerca de $70 \%$ superior ao o encontrado no presente estudo. Esse dado pode ser atribuído à análise do alho-poró inteiro feita pelos autores. No alho-poró tem sido descritos minerais como fósforo, ferro, potássio, cálcio, dentre outros. Quando comparado com a cebola, o mesmo apresenta maior quantidade de ferro (TACO, 2011). Minerais compõem um dos grupos de nutrientes necessários à manutenção da saúde, pois são componentes extremamente importantes ao metabolismo, e estão envolvidos em quase todas as reações e vias bioquímicas conhecidas (CUPPARI et al., 2004).

Com relação ao teor de proteínas, não houve diferença significativa quando comparadas as amostras entre si e suas partes (Tabela 1). Esses teores se assemelharam aos descritos por Storck et al. (2013) em talos de couve-flor $(1,56 \mathrm{~g} / 100 \mathrm{~g})$ e foram superiores em cerca de $13 \%$ aos 
descritos na tabela TACO (2011). A razão para essa diferença pode ser explicada pela análise de partes separadas da hortaliça.

Assim como as proteínas, o teor de gordura não apresentou diferença estatística entre as amostras de bulbo avaliadas, variando de $0,24 \pm 0,01$ a $0,25 \pm 0,01 \mathrm{~g} / 100 \mathrm{~g}$ (cultivares A e B, respectivamente; Tabela 1). No entanto, as amostras de folha apresentaram maior teor, embora sem diferença estatística entre os cultivares $(0,54 \pm 0,02$ versus $0,54 \pm 0,01 \mathrm{~g} / 100 \mathrm{~g})$. Storck et al. (2013) reportaram resultados semelhantes aos do presente estudo em folhas de couve-flor $(0,22 \mathrm{~g} / 100 \mathrm{~g})$. $\mathrm{O}$ conteúdo proteico descrito para a cebola (Allium cepa) é de $0,1 \mathrm{~g} / 100 \mathrm{~g}$. Em oposição, o alho (Allium sativum) apresenta teor de gordura $0,2 \mathrm{~g} / 100 \mathrm{~g}$, semelhante ao encontrado no bulbo do alho-poró (TACO, 2011). Em estudo realizado por Tsiaganis et al. (2006), que avaliou a composição de ácidos graxos dos lipídeos do gênero Allium, foi reportada a presença dos ácidos linoleico, palmítico, $\alpha$ - linolênico e oleico em maior quantidade na cebola, seguida do alho e alho-poró. Os teores de lipídeos da maioria das hortaliças são baixos, o que as torna, na maioria dos casos, de baixa densidade calórica.

No que se refere aos carboidratos, observou-se diferença significativa entre as amostras de bulbo avaliadas, sendo o teor do cultivar B cerca de $22 \%$ superior ao do A. A folha, por sua vez, apresentou menores teores quando comparada ao bulbo, com diferença entre as amostras, variando de 6,22 $\pm 0,75$ a 7,81 $\pm 1,24 \mathrm{~g} / 100 \mathrm{~g}$ (cultivares A e B, respectivamente; Tabela 1). Valores semelhantes aos encontrados na folha do alho-poró foram descritos por Storck et al. (2013) nas folhas de cenoura $(6,91 \mathrm{~g} / 100 \mathrm{~g})$ e na tabela TACO (2011) $(6,9 \mathrm{~g} / 100 \mathrm{~g})$. Dentre os carboidratos presentes nas hortaliças, destacam-se, por seu valor nutricional, as fibras. As fibras alimentares são um conjunto de substâncias derivadas de vegetais resistentes à ação das enzimas digestivas humanas (SCHWEIZER E EDWARDS, 1992). São classificadas em fibras solúveis, em grande parte constituída por pectinas, gomas e algumas hemiceluloses, e fibras insolúveis, principalmente celulose, hemiceluloses e lignina. De acordo com a resolução da ANVISA (2012) são considerados alimentos fontes de fibra os que apresentam no mínimo $3 \mathrm{~g} / 100 \mathrm{~g}$ e alimentos com alto conteúdo de fibras os que apresentam no mínimo $6 \mathrm{~g} / 100 \mathrm{~g}$. A partir dessa definição, pode-se classificar o alho-poró como um alimento com alto conteúdo de fibras, considerando que os carboidratos presentes no bulbo e folhas totalizam 16,3 e $20 \mathrm{~g} / 100 \mathrm{~g}$ (cultivares A e B, respectivamente).

Além das fibras, outra classe de compostos conhecidos como fitonutrientes, de elevada importância nutricional, são os polifenóis. Apesar de serem amplamente reconhecidos pelas suas atividades antioxidantes in vivo, os mesmos são considerados substâncias lábeis. Nesse sentido, objetivando avaliar a estabilidade dos teores de fenólicos totais e sua atividade antioxidante, as amostras foram processadas simulando manipulação doméstica (cozimento e refogado) e estes valores comparados a amostra in natura. Os teores de fenólicos totais estão apresentados na Tabela 2. 
Tabela 2 - Teor de fenólicos totais (mg EAG/100g) presentes em bulbo e folhas do alho-poró (Allium porrum).

\begin{tabular}{ccccc}
\hline \multicolumn{5}{c}{ Fenólicos totais } \\
\hline Partes & Cultivares & In natura & Refogado $^{*}$ & Cozido $^{\#}$ \\
\hline \multirow{2}{*}{ Bulbo } & A & $3,55 \pm 0,04^{\mathrm{b}}$ & $4,93 \pm 0,12^{\mathrm{a}}$ & $1,96 \pm 0,06^{\mathrm{c}}$ \\
& $\mathrm{B}$ & $4,34 \pm 0,07^{\mathrm{a}}$ & $3,61 \pm 0,37^{\mathrm{b}}$ & $2,00 \pm 0,05^{\mathrm{c}}$ \\
\multirow{2}{*}{ Folha } & $\mathrm{A}$ & $4,99 \pm 0,04^{\mathrm{b}}$ & $6,26 \pm 0,08^{\mathrm{a}}$ & $3,11 \pm 0,12^{\mathrm{c}}$ \\
& $\mathrm{B}$ & $5,55 \pm 0,06^{\mathrm{b}}$ & $6,46 \pm 0,25^{\mathrm{a}}$ & $3,59 \pm 0,43^{\mathrm{c}}$ \\
\hline
\end{tabular}

Legenda: *amostras foram refogadas utilizando uma colher de café de óleo de soja (6 g) até dourar; "fervura em água por 25 minutos, simulando um preparo de um caldo. EAG (equivalentes de ácido gálico). Valores resultantes da média de três repetições \pm desvio padrão. Em cada linha, letras diferentes diferem estatisticamente $(\mathrm{p}<0,05)$.

Os valores de fenólicos totais encontrados nas amostras in natura e processadas foram maiores nas amostras do cultivar B, tanto no bulbo quanto nas folhas (Tabela 2). Para o cultivar A, o conteúdo fenólico do bulbo foi inferior em cerca de $30 \%$ ao da folha, ambos in natura. Quando as amostras de bulbos foram refogadas, houve aumento, exceto para o cultivar B que teve redução de 17 \%. Adicionalmente, quando os bulbos foram cozidos, tanto o cultivar A quanto B tiveram seu conteúdo fenólico reduzido, sendo 45 e $54 \%$, respectivamente. O mesmo foi observado para as folhas na cocção, uma redução de 38 \% para o cultivar A e 35 \% para B. Por outro lado, quando refogadas, as folhas tiveram um aumento de 20 e 14\% na concentração fenólica (cultivares A e B, respectivamente). Analisando os dados em conjunto, pode-se constatar que, embora tenha havido uma pequena perda em uma amostra refogada, esse processo térmico foi capaz de contribuir no incremento do conteúdo fenólico total do alho-poró. Mais estudos são necessários, no entanto, para melhor elucidar estes achados.

Resultados encontrados por Najda et al. (2016) em estudo comparativo realizado com alho-porro-bravo (Allium ampeloprasum var. ampeloprasum) e alho (Allium sativum) mostraram-se inferiores aos achados do presente estudo. Em outro estudo realizado por Bernaert et al. (2012), que avaliou 30 variedades de alho-poró (Allium ampeloprasum var. porrum), os teores de fenólicos totais variaram de 5,31 a 13,96 mg GAE/100g, sendo o menor valor comparável com alguns resultados encontrados em nosso estudo. O teor fenólico total determinado por Bernaert et al. (2013) para o bulbo fervido por 20 minutos, foi significativamente menor quando comparado ao in natura, e, quando a fervura foi feita por uma hora, houve uma redução de 34 \%. Para a folha esta redução foi de 38 \%.

Afim de verificar se o processamento térmico interfere na capacidade redox do alho-poró, a sua atividade antioxidante foi mensurada (Tabela 3 ).

Tabela 3 - Atividade antioxidante (mM TE/g) de bulbo e folhas do alho-poró (Allium porrum).

\begin{tabular}{ccccc}
\hline \multicolumn{5}{c}{ Atividade antioxidante } \\
\hline Partes & Cultivares & In natura & Refogado & Cozido \\
\hline \multirow{2}{*}{ Bulbo } & A & $3,55 \pm 0,48^{\mathrm{a}}$ & $2,84 \pm 0,09^{\mathrm{b}}$ & $2,32 \pm 0,16^{\mathrm{b}}$ \\
& $\mathrm{B}$ & $4,34 \pm 0,27^{\mathrm{a}}$ & $4,47 \pm 0,61^{\mathrm{a}}$ & $2,82 \pm 0,02^{\mathrm{b}}$ \\
\multirow{2}{*}{ Folha } & A & $5,72 \pm 0,10^{\mathrm{b}}$ & $7,75 \pm 0,15^{\mathrm{a}}$ & $3,83 \pm 0,04^{\mathrm{c}}$ \\
& $\mathrm{B}$ & $7,10 \pm 0,22^{\mathrm{b}}$ & $10,26 \pm 0,14^{\mathrm{a}}$ & $3,54 \pm 0,55^{\mathrm{c}}$ \\
\hline
\end{tabular}

Legenda: TE (equivalentes de Trolox). Valores resultantes da média de três repetições \pm desvio padrão.

Em cada linha, letras diferentes diferem estatisticamente $(p<0,05)$. 
Os resultados encontrados indicam um comportamento semelhante ao observado para os fenólicos totais, sendo maior no cultivar B, tanto no bulbo quanto nas folhas in natura. É possível observar também que a atividade antioxidante para ambos os cultivares foi maior nas folhas do que nos bulbos (uma diferença média de $20 \%$ para amostras in natura). Outro achado relevante é que o cozimento foi capaz de reduzir significativamente esta atividade nos dois cultivares, tendo sido observada uma redução máxima de 50 \% para as folhas do cultivar B. Quando refogadas, no entanto, as amostras tiveram sua atividade antioxidante aumentada, exceto para os bulbos do cultivar A, que exibiram uma redução de $20 \%$. Dessa forma, verifica-se que o tempo e forma de processamento térmico tem grande influência na redução ou aumento da atividade antioxidante. No que se refere à redução da atividade, esta pode ser justificada pela instabilidade térmica dos polifenóis em solução aquosa, como previamente observado (BERNAERT et al., 2013). Por outro lado, um dos motivos para o aumento pode ser explicado pela preservação de compostos com $\beta$-caroteno, luteína e vitamina $\mathrm{E}$, dentre outros compostos lipofílicos com atividade antioxidante (GAYATHRI et al., 2004; KURZ et al., 2008). Não existem muitos estudos acerca da atividade antioxidante do alho-poró. Previamente foi reportado que o mesmo apresenta atividade superior à relatada para a alho comum, sendo 81,14 $\mu \mathrm{mol} \mathrm{TE} / \mathrm{g}$ e 67,23 $\mu \mathrm{mol} \mathrm{TE} / \mathrm{g}$, respectivamente. Nossos achados estão de acordo com os encontrados por Bernaert et al. (2013), os quais descreveram redução da atividade antioxidante para o alho-poró cozido por 20 minutos, no entanto, quando as folhas passaram por branqueamento, houve incremento de $19 \%$ na capacidade antioxidante. Ainda, os autores reportaram aumento após fervura por 40 ou 60 minutos de 12 e $21 \%$ respectivamente, pós branqueamento. É importante destacar que não há consenso na literatura quanto à temperatura ótima para extração dos polifenóis. Por exemplo, no estudo de Ye et al. (2014), foi mostrado que as condições ideais de extração foram $80^{\circ} \mathrm{C}$ por $5 \mathrm{~h}$. Por outro lado, Cvetanović et al. (2019) relataram uma temperatura de $150{ }^{\circ} \mathrm{C}$ como ótima para a atividade antioxidante de um extrato rico em polifenóis. Além disso, foi observado que dependendo da estrutura do composto fenólico e de sua conjugação com açúcares, ésteres, hidroxilas ou amidas, as condições de extração mudam porque essas moléculas influenciam a eficiência da extração de polifenóis e, consequentemente em sua atividade antioxidante. Essas evidências indicam que a alteração (aumento/redução) no conteúdo fenólico total é também dependente da matriz química da amostra vegetal em questão. Dessa forma, mais estudos são necessários para monitorar a oscilação desses parâmetros no Alho-poró em função do tempo e temperatura.

Aproximadamente um terço do alimento produzido no mundo é desperdiçado, o que equivale a 1,3 bilhão de toneladas anuais. Em países como os Estados Unidos, Austrália e Inglaterra, o percentual descartado ultrapassa um terço da produção. Esses países concentram a maior parte do desperdício no final da cadeia de produção. Nos países em desenvolvimento, o desperdício também é elevado e as perdas tendem a ser grandes desde o manejo da lavoura e o pós-colheita até o consumidor final (EMBRAPA, 2018). Cascas e folhas geralmente são descartadas, porém estes podem agregar enriquecimento alimentar, aumentando o valor nutricional das refeições, já que são partes frequentemente 
descritas como ricas em nutrientes comparativamente com as partes nobres (SOUZA et al., 2007). No presente estudo foi observado que a folha do alho-poró apresenta maiores quantidades de minerais, fenólicos totais e atividade antioxidante quando comparada ao bulbo, tradicionalmente reconhecida como a parte nobre dessa hortaliça. Esse achado está de acordo com estudos prévios que demonstraram um conteúdo fenólico superior nas folhas do que em partes comestíveis de frutos, como maçã, marmelo, groselha e até mesmo o mirtilo (TELESZKO E WOJDYŁO, 2015). Os polifenóis se distribuem de forma heterogênea nas diferentes partes do vegetal, sendo que as maiores concentrações são observadas nas partes mais expostas à radiação solar, como as folhas e cascas dos frutos (GONDIM et al., 2005; MARQUES et al., 2010; HUA et al., 2018). Essas são evidências anteriores que poderiam ajudar a compreender porque as folhas do Alho-poró concentram mais polifenóis do que o bulbo. Dessa forma, nossos achados reforçam a importância do consumo integral do alho-poró.

\section{CONCLUSÃO}

O alho-poró é um alimento com grande aplicabilidade na culinária. Suas características, como versatilidade e o sabor aromático marcante o tornam um extraordinário ingrediente que agrega, não somente sabores e texturas às preparações, mas também macro, micro e fitonutrientes. $\mathrm{O}$ presente estudo demonstrou que o alho-poró do cultivar Lancelot apresentou conteúdo de umidade, cinzas, lipídeos e polifenóis totais superior ao cultivar Atal, assim como uma maior capacidade antioxidante. Independentemente da variedade, observou-se que as melhores formas para o consumo do alho-poró são in natura ou refogado, pois assim pode-se conservar e mesmo aumentar seu conteúdo fenólico e, consequentemente, sua capacidade antioxidante. Por ser produzido em nível mundial, e ser facilmente adaptável aos diferentes climas e solos, seu consumo deve ser incentivado e valorizado. Por fim, por se tratar de uma hortaliça rica em minerais e polifenóis, e tendo todas as suas partes comestíveis, é importante priorizar o seu consumo de forma integral, evitando o desperdício.

\section{REFERÊNCIAS}

ANVISA. AGENCIA NACIONAL DE VIGILÂNCIA SANITÁRIA. Resolução da diretoria colegiada RDC n 54, de 12 de novembro de 2012. Disponível em: https://bit.ly/3pmdhTs. Acesso em: 03 mar. 2020.

AOAC. Official Methods of Analysis of AOAC International. 18. Ed., AOAC International, Gaitherburg, $2590,2011$.

ASEMANI, Y. et al. Allium vegetables for possible future of cancer treatment. Phytotherapy Research, v. 33, n. 12, p. 3019-3039, 2019. 
BERNAERT, N. et al. Antioxidant capacity, total phenolic and ascorbate content as a function of the genetic diversity of leek (Allium ampeloprasum var. porrum). Food Chemistry, v. 134, n. 2, p. 669-677, 2012.

BERNAERT, N. et al. Antioxidant changes during domestic food processing of the white shaft and green leaves of leek (Allium ampeloprasum var. porrum). Journal of the Science of Food and Agriculture, v. 94, n. 6, p. 1168-1174, 2013.

BLIGH, E. G.; DYER, W. J. A rapid method of total lipid extraction and purification. Canadian Journal of Biochemistry and Physiology, v. 37, n. 8, p. 911-917, 1959.

CLERCQ, H. et al. Causal relationships between inbreeding, seed characteristics and plant performance in leek (Allium porrum L.). Euphytica, v. 1, n. 134, p. 103-115, 2003.

CORREAA, M. P. Dicionário das plantas úteis do Brasil e das exóticas cultivadas. Rio de Janeiro: Imprensa Nacional, v. 6, 1978.

CUPPARI, L. et al. Preparo de vegetais para utilização em dieta restrita em potássio. Brazilian Society for Food and Nutrition, v. 8, n. 2, p. 1-7, 2004.

CVETANOVIĆ, A. et al. The influence of the extraction temperature on polyphenolic profiles and bioactivity of chamomile (Matricaria chamomilla L.) subcritical water extracts. Food Chemistry, v. 27, p. 328-337, 2019.

EMBRAPA. EMPRESA BRASILEIRA DE PESQUISA AGROPECUÁRIA. Perda e desperdício de alimentos. Disponível em: https://bit.ly/3nKm51H. Acesso em: 18 jun. 2018.

GAYATHRI, G. N. et al. Influence of antioxidant spices on the retention of $\beta$-carotene in vegetables during domestic cooking processes. Food Chemistry, v. 84, n. 1, p. 35-43, 2004.

GONDIM, J. A. M. et al. Composição centesimal e de minerais em cascas de frutas. Ciência e Tecnologia de Alimentos, v. 25, n. 4, p. 825-827, 2005.

HSING, A. et al. Allium vegetables and risk of prostate cancer: a population-based study. Cancer Spectrum Knowledge Environment, v. 94, n. 21, p. 1648-1651, 2002. 
HUA, Q. et al. Metabolomic characterization of pitaya fruit from three red-skinned cultivars with different pulp colors. Plant Physiology and Biochemistry, v. 126, p. 117-125, 2018.

IAL. INSTITUTO ADOLFO LUTZ. Métodos físico-químicos para análise de alimentos. São Paulo: Instituto Adolfo Lutz, 1020 p, 2008.

KOTHARI, D. et al. The genus allium as poultry feed additive: a review. Animals, v. 9, n. 12, p. 1032, 2019.

KURZ, C; et al. HPLC-DAD-MS in characterisation of carotenoids from apricots and pumpkins for the evaluation of fruit product authenticity. Food Chemistry, v. 110, n. 2, p. 522-530, 2008.

LANZOTTI, V. The analysis of onion and garlic. Journal of Chromatography A, v. 1112, n. 1-2, p. 3-22, 2006.

MARQUES, A. et al. Composição centesimal e de Minerais de casca e polpa de manga (Mangifera indica L.) cv. Tommy Atkins. Revista Brasileira de Fruticultura, v. 32, n. 4, p. 1206-1210, 2010.

NAJDA, A. et al. Comparative studies of nutritional and health-enhancing properties in the "garlic-like" plant Allium ampeloprasum var. Ampeloprasum (GHG-L) and A. sativum. Scientia Horticulturae, v. 201, p. 247-255, 2016.

NICASTRO, H.L. et al. Garlic and onions: their cancer prevention properties. Cancer Prevention Research, v. 8, n. 3, p. 181-9, 2015.

PAK, L. M. et al. Avaliação da composição nutricional do alho-porro (Allium porrum, Aliaceae). Visão Acadêmica, v. 15, n. 3, p. 51-66, 2014.

PROTEGGENTE, A. et al. The antioxidant activity of regularly consumed fruit and vegetables reflects their phenolic and vitamin C composition. Free Radical Research, v. 2, n. 36, p. 217-233, 2002.

RE, R. et al. Antioxidant activity applying an improved ABTS radical cation decolorization assay. Free Radical Biology \& Medicine, v. 26, n. 9-10, p. 1231-1237, 1999.

SCHWEIZER, T.F.; EDWARDS, C. A. Dietary fibre: a component of food nutritional function in health and disease. London: Springer Verlag, p. 57-99. 1992. 
SHAD, A. A. et al. Antioxidant activity and nutritional assessment of under-utilized medicinal plants.

Pakistan Journal of Pharmaceutical Sciences, p. 2039-2045, 2016.

SHARIFI-RAD, J. et al. Plants of the genus Allium as antibacterial agents: from tradition to pharmacy. Cellular and Molecular Biology, v. 62, n. 9, p. 57-68, 2016.

SINGLETON, V.L.; ROSSI, J. A. Colorimetry of total phenolics with phosphomolybdic phosphotungstic acid reagents. American Journal of Enology and Viticulture, n. 16, p. 144-158, 1965.

SOUZA, P.D.J. et al. Analise sensorial e nutricional de torta salgada elaborada através do aproveitamento alternativo de talos e cascas de hortaliças. Alimentação e Nutrição, v. 18, n. 1, p. 5560, 2007.

STORCK, C. R. et al. Folhas, talos, cascas e sementes de vegetais: composição nutricional, aproveitamento na alimentação e análise sensorial de preparações. Ciência Rural, v. 43, n. 3, p. 537-546, 2013.

TACO. Tabela Brasileira de Composição de Alimentos. 4. Ed. Revisada e Ampliada. Campinas: NEPA- UNICAMP, 161 p., 2011.

TELESZKO, M., WOJDYŁO, A. Comparison of phenolic compounds and antioxidant potential between selected edible fruits and their leaves. Journal of Functional Foods, v. 14, p. 736-746, 2015.

TSIAGANIS, M. C. et al. Fatty acid composition of Allium species lipids. Journal of Food Composition and Analysis, v. 19, n. 6-7, p. 620-627, 2006.

YE, Y. et al. Optimization of phenolics extracted from Idesia polycarpa defatted fruit residue and its antioxidant and depigmenting activity in vitro and in vivo. Evidence-Based Complementary and Alternative Medicine, Article ID 931269, 2014. 